\title{
Estimation of the Probability of Congestion using Monte Carlo method in OPS Networks*
}

\author{
Anna Urra ${ }^{\dagger}$, Jose L. Marzo, Mateu Sbert ${ }^{\ddagger}$, Eusebi Calle \\ Institute of Informatics and Applications (IIiA) \\ University of Girona, Avinguda Lluis Santalo s/n, 17071 Girona, Spain \\ \{aurra,marzo\}@eia.udg.es, mateu@ima.udg.es, eusebi@eia.udg.es
}

\begin{abstract}
In networks with sniall buffers, such as Optical Packet Switching based networks, the Convolution Approach is presented as one of the most accurate method used for the Connection Admission Control. Admission control and resource management have been addressed in other works oriented to bursty traffic and ATM. This paper focuses on heterogeneous traffic in OPS based networks. Using heterogeneous traffic and bufferless networks the Enhanced Convolution Approach is a good solution. However, both methods (CA and ECA) present a high computational cost for high number of connections. Two new mechanisms (UMCA and ISCA) based on Monte Carlo method are proposed to overcome this drawback. Simulation results show that our proposals achieve lower computational cost compared to Enhanced Convolution Approach with an small stochastic error in the probability estimation.
\end{abstract}

\section{Introduction}

One of the dominant solutions for the next generation Internet is expected to be optical technologies $[1,2]$. In this context, bandwidth management and traffic control strategies are necessaries in order to allow for high utilization of network resources, whilst sustaining and acceptable QoS for all connections. This paper studies the provision of guaranteed QoS in terms of probability of congestion into an IP over Optical Packet Switching (OPS) network scenario. Due to the use of OPS, delay is also limited by the utilization of small buffers in switches.

Internet traffic is aggregated and carried over optical core

${ }^{*}$ This work was supported by the Spanish Research Council (CICYT) under contract TIC2003-05567.

tThe work of Anna Urra was supported by the Ministry Universities, Research and Information Society (DURSI) and European Social Funds.

$\ddagger$ The work of Mateu Sbert was supported by the CICYT under contract TIN2004-07451-C03-01. networks. However, QoS mechanisms proposed in IP networks do not have an easy deployment in optical networks. This is the case of implementating of packet queues for packet contention resolution. Contention may arise when two or more packets demand the utilization of the same resources, for instance, when two or more packets have to be forwarded to the same output link at the same time. In traditional electronic packet switches, packet queues are implented using buffers. Nevertheless, buffers to implement optical packet queues do not exist in a similar way. In optical networks Fiber Delay Lines (FDLs) are used to implement optical packet queues. FDLs are long fiber lines used to delay the optical signal for a particular period of time in order to simulate a buffer.

Kaheel [1] presents a classification of the major optical switching methods: Wavelength Routing (WR), Optical Packet Switching (OPS) and Optical Burst Switching (OBS). WR presents low bandwidth utilization because it does not use statistical sharing of resources [1]. On the other hand, OPS and OBS networks where packets streams can be multiplexed statistically, making a more efficient use of capacity [2].

This work focuses on OPS networks where statistical multiplexing for bandwidth sharing is used. In addition, OPS offers fast allocation of channels with fine granularity, high-speed and configurability [2]. These are some of the important main features needed in the future networks to support different forms of data [2]. Figure 1 shows the optical architecture. Packets are aggregated at the edge of the network reducing the processing overhead, and then are routed over a bufferless core network (OPS).

Although admission control and resource management have been addressed in other works [3, 4], most of them are oriented to bursty traffic. However, our proposal focuses on heterogeneous traffic.

The paper is organized as follows. Section 2 presents basic notions related to $\mathrm{CAC}$ and statistical multiplexing gain. Proposed mechanisms to evaluate the Probability of Congestion are reviewed and two new mechanisms are pro- 


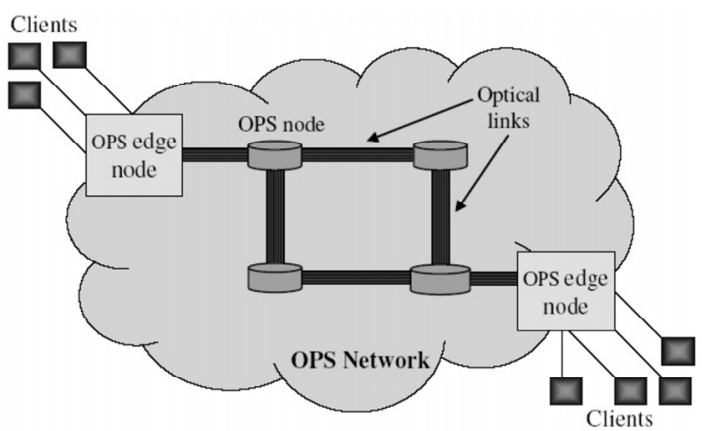

Figure 1. Optical Architecture

posed in section 3 . The simulations and performance results are presented in section 4 . Section 5 concludes the paper.

\section{Traffic and Congestion Control}

In this section the Connection Admission Control (CAC) as a traffic and congestion control function is reviewed. Statistical multiplexing gain, one of the requirements in a CAC procedure, is also reviewed. In addition, different aproximations to evaluate the banwidth demanded are depicted.

\subsection{Connection Admission Control}

In order to avoid congestion, different traffic control methods exists. Traffic control methods can be divided in two categories: reactive control and preventive control. $R e$ active control methods regulate the traffic flow at the access points based on current traffic levels within the network. Preventive control methods provide a fair allocation of bandwidth by requiring, at times of high network load, that each connection's traffic flow remains within specified bounds appropriate for the supported service [5]. Due to real time constraints preventive control is more suitable than reactive control in high-speed networks. Connection $A d-$ mission Control (CAC) is one of the traffic and congestion preventive control function used to maintain the QoS requested. CAC is the procedure responsible for determining whether a connection request is accepted or rejected. The main requirements in a $\mathrm{CAC}$ can be summarized as follows:

- The network must be protected from overload.

- Resources must be allocated in such a way that the QoS requirements are met for all established connections.

- Maximal statistical multiplexing gain should be obtained.

- The required real-time processing should be reasonable.

Maximal statistical multiplexing gain can be achieved if the network knows the probability density function of the individual sources.

\subsection{Statistical Multiplexing Gain}

Efficiency gain depends on the statistical multiplexing effect of sources, with the condition that enough sources are multiplexed and that they are not correlated. Statistically multiplexing packet loss probability and delay performance depend on link utilization and buffer size, as well as traffic characteristics of the connection on the link. This assumption is not always fulfilled; neither is it an easy job to calculate the convolution at every link where different sources are multiplexed. There are some approximations to evaluate the bandwidth demanded by a set of connections:

- Linear CAC. It reduces the CAC task to the simple problem of determining whether the sum of the effective bandwidth of each of the connections is greater than the resource capacity; if that is the case the connection is rejected, otherwise it is accepted.

- Fluid Flow approximation. This model assumes that the information arrives uniformly during a burst and that the server removes the information from the queue in the same manner. In general, this model is valid when the buffer capacity is longer than the mean burst duration.

- Stationary approximations. In this case the effect of statistical multiplexing is the dominant factor. It considers that packets are lost when the instantaneous rate is greater than the bandwidth provided by the link. Stationary approaches are Binomial, Gaussian and Convolution.

- Heuristic methods. Heuristic approaches provide a mechanism for clustering data obtained from traffic measurements in a structure that constitutes the traffic model. The neural network and fuzzy logic based approaches are examples of this kind of approaches [6].

In small buffer networks, the Convolution Approach (CA) is the most accurate method used in CAC. But it has a considerable computational cost and a high number of accumulated calculations. Nevertheless, in critical near-congestion situations, the convolution is the only algorithm that gives enough accuracy. CAC has been widely studied especially in the context of ATM networks $[7,8,9]$. In our previuos work [7], we proposed and Enhanced Convolution Approach (ECA) that reduces the computational cost in CA. A review of these methods is presented in next sections in order to adapt them to OPS networks and new calculation mechanisms based on Monte Carlo techniques (Uniform Monte Carlo and Importance Sampling) are proposed.

\section{Calculation of the Bandwidth Requirements}

In this section different methods used to compute the bandwidth requirements of the superposition of several sources, including our proposals, are described. 


\subsection{Model Formalization}

Let suppose that there are $t$ types of sources where each source $s$ emits in $m_{s}$ states. Each state- $i$ has an associated rate $r_{i}^{s}$ and probability $p_{i}^{s}$.

For each source $s$, there are $n_{s}$ connections, then $c_{i}^{s}$ connections are in state $i$. Therefore:

$$
\sum_{i=0}^{m_{s}-1} c_{i}^{s}=n_{s}, \quad \forall s
$$

Link has a maximum capacity avalaible, $C_{\max }$. Congestion is produced when the addition of connection rates is greater than $C_{\max }$.

Figure 2 shows an example of the model used. Twotypes of sources are considered. Source $s_{0}$ and $s_{1}$ have 2 $\left(m_{0}\right)$ and $3\left(m_{1}\right)$ states, respectively. State 2 of source 1 has an emission rate of $r_{2}^{1}$ units with a probability of $p_{2}^{1}$. There are $n_{0}$ connections of type 0 and $n_{1}$ connections of type 1 .

\subsection{Convolution Approach}

The Convolution Approach (CA) is based on the convolution expression:

$$
P(Y+X=b)=\sum_{k=0}^{b} P(Y=b-k) P(X=k) .
$$

where $Y$ is the bandwidth requirement of the already established connections; $X$ is the bandwidth requirement of a new connection request, and $b$ denotes the instantaneous required bandwidth. In fact the CA obtains a probability density funcion for the offered system load, expressed as the probability that all traffic sources together are emitting at a giving rate. The size of the storage [10] required presents a dimensioning problem. This requirement increases with the number of connections and possible source states. In addition, the probability is the result of a large number of previous calculations.

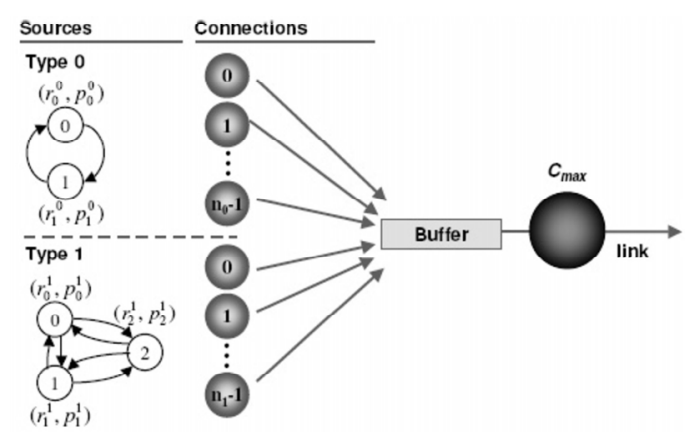

Figure 2. Model architecture sample

\subsection{Enhanced Convolution Approach}

In our previous work [7], we have proposed a faster method for overcoming the CA drawbacks: the Enhanced Convolution Approach (ECA). This is carried out by applying the Multinomial Distribution Function (MDF) [11].

Initially, we assume only one type of source $s$ emitting in $m_{s}$ states (homogeneous traffic). We can consider a $m_{s}$ dimensional random variable $\left(e_{0}^{s}, e_{1}^{s}, \ldots, e_{m_{s}-1}^{s}\right)$ for each source $s$. Therefore a random event that has been repeated $n_{s}$ times (considering all the connections at a time) has the characteristic $\left(c_{0}^{s}, c_{1}^{s}, \ldots, c_{m_{s}-1}^{s}\right)$. It is necessary to calculate the probability of $e_{0}^{s}$ occurring $c_{0}^{s}$ times, $e_{m_{s}-1}^{s}$ occurring $c_{m_{s}-1}^{s}$ times. For this purpose the generalized Bernoulli trials are considered. As in the previous situation, the probability

$$
\left(p_{0}^{s}\right)^{c_{0}^{s}} \cdot\left(p_{1}^{s}\right)^{c_{1}^{s}} \ldots\left(p_{m_{s}-1}^{s}\right)^{c_{m_{s}-1}^{s}}
$$

is assigned to the point $\left(e_{0}^{s}, e_{1}^{s}, \ldots, e_{m_{s}-1}^{s}\right)$ with $\left(c_{0}^{s}, c_{1}^{s}, \ldots, c_{m s_{-} 1}^{s}\right)$ connections.

This is the probability assigned to any specific sequence having $c_{i}^{s}$ occurrences of $e_{i}^{s}$ varying $i=0,1, \ldots, m_{s}-1$. Thus, the number of sequences having exactly $c_{0}^{s}$ connections in state $e_{0}^{s}, c_{1}^{s}$ connections in state $e_{1}^{s}, \ldots$, and $c_{m_{s}-1}^{s}$ connections in state $e_{m_{s}-1}^{s}$ is:

$$
\frac{n_{s} !}{c_{0}^{s !} c_{1}^{s ! \ldots c_{m_{s}-1}^{s} !}}
$$

Finally, the probability of this characteristic is, according to [7]:

$P$ (state $e_{0}^{s}$ occurs $c_{0}^{s}$ times, $\ldots$, state $e_{m_{s}-1}^{s}$ occurs $c_{m_{s}-1}^{s}$ times $)=$

$$
\frac{n_{s} !}{c_{0}^{s} ! c_{1}^{s} ! \ldots c_{m_{s}-1}^{s} !}\left(p_{0}^{s}\right)^{c_{0}^{s}} \cdot\left(p_{1}^{s}\right)^{c_{1}^{s}} \ldots\left(p_{m_{s}-1}^{s}\right)^{c_{m_{s}-1}^{s}}
$$

Note that the probability of each source beginning in the state $e_{i}^{s}$ is independent of the probability of the other sources.

The multinomial probability distribution is applied to groups of the same type of sources (homogeneous traffic), and the general state probabilities are evaluated by convolution of the partial results obtained from the different existing groups of sources (heterogeneous traffic).

The decision criterion in order to accept a new connection of $j$-type when the ECA is used in CAC is:

$$
\begin{gathered}
P C\left(Y+X_{j}\right)=P\left(Y+X_{j}>\gamma \cdot C_{\max }\right)= \\
\sum_{b>\gamma \cdot C} P\left(Y+X_{j}=b\right)<\epsilon
\end{gathered}
$$

Where $P C$ is the Probability of Congestion; $Y$ is the Bandwidth requirement of the already established connections; 
$X_{j}$ is the bandwidth requirement of a new $j$-type connection; $b$ is the instantaneous rate considered; $\epsilon$ is the maximum accepted Probability of Congestion due to excess bandwidth required, set according to the QoS requirements; and $\gamma$ is the load factor that one has to pay regard to in order to limit congestion arising from contention resolution on the packet level. The maximum load is restricted to a predefined value $\gamma$. This has to be taken care of by a proper choice of $\gamma$. For the same reason the bandwidth provided by the link, and not the capacity of the link, determines the limit of excess.

Figure 3 shows the area evaluated using $\mathrm{CA}$ and ECA. In $\mathrm{CA}$ case (Fig. $3 \mathrm{a}$ ), all the probabiliy density function is computed. On the other hand, in ECA case (Fig. 3b) only the probability density function with final rate higher than $C_{\max }$ is computed reducing the computational cost. ECA also uses other mechanisms that reduce the computational cost as cut-off mechanisms [7].

\subsection{Estimation of the Probability of Congestion us- ing Monte Carlo Methods}

This section describes the two proposed mechanisms based on Monte Carlo methods. In this section we assume all connection are not grouped according to the same type of sources. Therefore, we consider $n$ as the number of connections, i.e., number of sources. Note that $\sum_{i=0}^{l} n_{i}=n$.

1. Uniform Monte Carlo Convolution Approach (UMCA). Suppose we have $n$ sources, where each source $s$ is characterized by the random variable $r^{s}$ with emission rates $\left\{r_{1}^{s}, r_{2}^{s}, \ldots, r_{i}^{s}, \ldots, r_{m_{s}}^{s}\right\}$ with probabilities $\left\{p_{1}^{s}, p_{2}^{s}, \ldots, p_{i}^{s}, \ldots, p_{m_{s}}^{s}\right\}$. The expected value of a function of the $n$ random variables $r^{s}$ is then given by:

$$
\mathbf{E}\left(f\left(r^{1}, \ldots, r^{n}\right)\right)=\sum_{i_{1}, \ldots, i_{n}} f\left(r_{i_{1}}^{1}, \ldots, r_{i_{n}}^{n}\right) p_{i_{1}}^{1} \cdots p_{i_{n}}^{n}
$$

We can evaluate with the Monte Carlo method [12] the sum (7). We have to select a joint probability distribution $\left\{\mathbf{q}^{1}, \ldots, \mathbf{q}^{n}\right\}$, where $\mathbf{q}^{s}=$

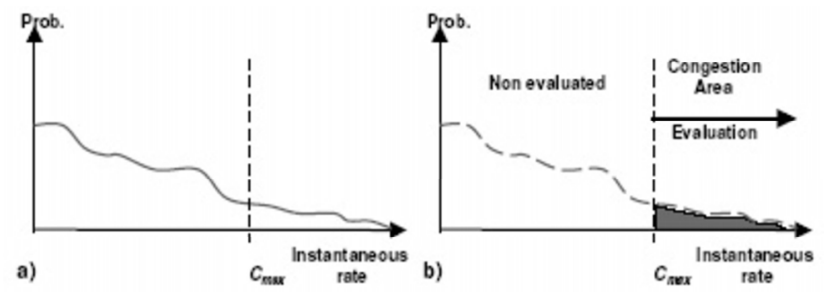

Figure 3. Probability of Congestion evaluation using a) Convolution Approach b) Enhanced Convolution Approach $\left\{q_{1}^{s}, q_{2}^{s}, \ldots, q_{i}^{s}, \ldots, q_{m_{s}}^{s}\right\}$, and sample from this joint distribution, which can be done by sampling independently from each $\mathbf{q}^{s}$. Given $N$ samples from $\left\{\mathbf{q}^{1}, \ldots, \mathbf{q}^{n}\right\},\left\{q_{j}^{1} \cdots q_{j}^{n}\right\}, 1 \leq j \leq N$, the estimator for the sum (7) will be:

$\mathbf{E}\left(f\left(r^{1}, \ldots, r^{n}\right)\right) \approx \frac{1}{N} \sum_{j=1}^{N} \frac{f\left(r_{j}^{1}, \ldots, r_{j}^{n}\right) p_{j}^{1} \cdots p_{j}^{n}}{q_{j}^{1} \cdots q_{j}^{n}}$

A particular case is selecting $\left\{\mathbf{q}^{1}, \ldots, \mathbf{q}^{n}\right\}=$ $\left\{\mathbf{p}^{1}, \ldots, \mathbf{p}^{n}\right\}$, and estimator (8) becomes

$$
\mathbf{E}\left(f\left(r^{1}, \ldots, r^{n}\right)\right) \approx \frac{1}{N} \sum_{j=1}^{N} f\left(r_{j}^{1}, \ldots, r_{j}^{n}\right)
$$

2. Importance Sampling Convolution Approach (ISCA). Estimator (9) is a cheap alternative in terms of computing cost, but it can give a high error in the estimation if the value of the function $f$ is not evenly distributed. As we are free to select the $\mathbf{q}^{s}$ probabilities a good alternative, known as importance sampling technique [12], is to select these probabilities proportional to each term in the sum, i.e.,

$$
q_{j}^{1} \cdots q_{j}^{n} \propto f\left(r_{j}^{1}, \ldots, r_{j}^{n}\right) \cdot p_{j}^{1} \cdots p_{j}^{n}
$$

We are interested in evaluating the expected value of boolean function $f\left(r^{1}, \ldots, r^{n}\right)=\left(\sum_{j=1}^{n} r^{j}>\right.$ $C_{\max }$ ), where $C_{\max }$ is the maximum capacity of the channel. This is, we are interested in estimating the probability of channel congestion. Selecting probabilities as in expression (10) is not practical for a large number of sources, as the cost of its evaluation grows combinatorially. What we will do is to assign a higher probability to the highest values $V$. A further assignment for all other values of probability $q$ proportional to $p$ would allow for a cheap evaluation of quotients $\frac{p}{q}$.

\section{Performance Evaluation}

In this section a complete set of experiments in order to prove the improvement in performance of the proposed methods are explained.

\subsection{Model Adopted}

For this set of experiments, we consider 5-types of sources. Table 1 shows the associated emission rates and probabilities of each source.

In order to evaluate mechanism performances we also consider four scenarios varying proportionally the number of connections (see Table 2). 
Table 1. Rates and Probabilities of the Sources

\begin{tabular}{|c|c|c|c|c|c|}
\hline$s$ & $r$ & $p$ & $s$ & $r$ & $p$ \\
\hline \multirow[t]{3}{*}{1} & 5 & 0.125 & \multirow[t]{3}{*}{4} & 5 & 0.145 \\
\hline & 10 & 0.645 & & 10 & 0.731 \\
\hline & 20 & 0.23 & & 25 & 0.124 \\
\hline \multirow[t]{2}{*}{2} & 5 & 0.885 & \multirow[t]{3}{*}{5} & 5 & 0.333 \\
\hline & 25 & 0.115 & & 15 & 0.601 \\
\hline \multirow[t]{2}{*}{3} & 0 & 0.895 & & 30 & 0.066 \\
\hline & 15 & 0.105 & & & \\
\hline
\end{tabular}

Table 2. Number of connections $(n)$ for each scenario

\begin{tabular}{|c|c|c|c|c|c|c|}
\hline \multirow[t]{2}{*}{ Scenario } & \multicolumn{5}{|c|}{ Type of Sources } & \multirow[t]{2}{*}{$n$} \\
\hline & $n_{1}$ & $n_{2}$ & $n_{3}$ & $n_{4}$ & $n_{5}$ & \\
\hline A & 10 & 9 & 11 & 5 & 2 & 37 \\
\hline $\mathrm{B}$ & 20 & 18 & 22 & 10 & 4 & 74 \\
\hline $\mathrm{C}$ & 30 & 27 & 33 & 15 & 6 & 111 \\
\hline $\mathrm{D}$ & 40 & 36 & 44 & 20 & 8 & 148 \\
\hline
\end{tabular}

As explained in section 3.4, ISCA assigns a higher probability to the highest rates of each source. For this set of experiments we assign a probability of 0.5 to the highest rate of each source. The rest of rates have a probability equal to $\frac{1-0.5}{m_{s}-1}$.

\subsection{Figures of Merit}

To evaluate the mechanism performances, three figures of merit are used in the experiments:

1. Computational cost. The drawback of using ECA is the resulting computational cost when a high number of connections must be considered. This parameter is evaluated in different sets of experiments in terms of time (seconds) for ECA, UMCA and ISCA. All mechanisms are implemented on a Linux machine with a Pentium-4 2.60 GHz processor using the programming language $\mathrm{C}$.

2. Absolute error. UMCA and ISCA are an alternative to compute the Probablity of Congestion, but they can give an error in the estimation. The absolute error is also considered in order to evaluate the performance of both mechanisms.

3. Relative efficiency. Although the error produced on the estimation using UMCA is higher than ISCA, it results on better computational cost. Comparison between two estimators given by probabilities $\mathbf{q}^{s, 1}$ and $\mathbf{q}^{s, 2}$ is done in terms of efficiencies. If $\mathbf{V}_{\mathbf{1}}, \mathbf{V}_{\mathbf{2}}, t_{1}, t_{2}$ are the respective variances (or expected quadratic errors) and time cost, then relative efficiency of the two estimators is defined as the quotient

$$
\frac{\mathrm{V}_{1} \times t_{1}}{\mathrm{~V}_{2} \times t_{2}}
$$

\subsection{Simulation Results}

\section{1) Computational Cost}

Table 3 shows the computational cost in terms of time (s) varying the maximum capacity of the channel $\left(C_{\max }\right)$. This experiment has been computed over scenario D (high number of connections). ISCA and UMCA have been computed using 100.000 iterations and they show similar behaviour for all cases (around 5.17s and 4.5s respectively). On the other hand, ECA decreases the computational time as $C_{\max }$ increases (see section 3.3). For instance as shown in Table 3 , the computational cost is equal to $3625 \mathrm{~s}$ in the case of $C_{\max }=1700$ and it is equal to 2391 in the case of $C_{\max }=1800$. Therefore, there is a computational cost decrease of around $34 \%$. However, ECA always offers a higher computational cost than UMCA and ISCA. Next ex-

Table 3. Computational Cost (seconds) varying $C_{\max }$

\begin{tabular}{|c||c|c|c|}
\hline Cmax & ECA & UMCA & ISCA \\
\hline 900 & 7631 & 4,5 & 5,17 \\
\hline 1000 & 7503 & 4,5 & 5,17 \\
\hline 1100 & 5019 & 4,5 & 5,17 \\
\hline 1200 & 5902 & 4,5 & 5,17 \\
\hline 1300 & 6629 & 4,5 & 5,17 \\
\hline 1400 & 6259 & 4,5 & 5,13 \\
\hline 1500 & 5305 & 4,5 & 5,13 \\
\hline 1600 & 4492 & 4,5 & 5,17 \\
\hline 1700 & 3625 & 4,5 & 5,17 \\
\hline 1800 & 2391 & 4,5 & 5,13 \\
\hline
\end{tabular}

periment evaluates the computational cost of ECA, UMCA and ISCA for each scenario A, B, C, D. Figures $4 \mathrm{a}$ and $4 \mathrm{~b}$ show the computational cost obtained considering 100.000 and 500.000 iterations, respectively. UMCA is the faster mechanism in terms of computational cost (Fig. 4a). However, when the number of iterations increases, both UMCA and ISCA have a computational cost higher than ECA for low number of connections (see Fig. 4b).

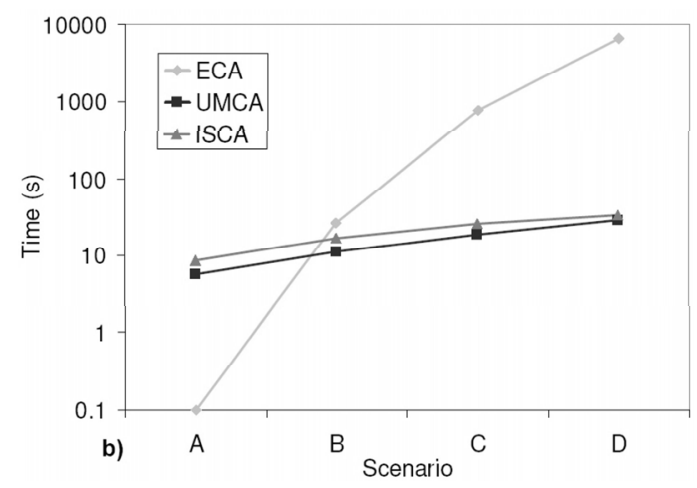

Figure 4. Computational cost a) 100.000 iterations b) 500.000 iterations 


\section{2) Absolute Error}

The absolute error of the Probability of Congestion (PC) is expressed as the difference between the estimated value (UMCA and ISCA) and the exact value (ECA). Now, we evaluate Probability of Congestion of UMCA and ISCA for scenario $A$ varying the number of iterations. Figure 5 shows PC of ISCA performs in a similar way to ECA than UMCA. Therefore, as expected, the absolute error of UMCA is higher than ISCA. In addition, the large is the number of iterations, the less is the absolute error.

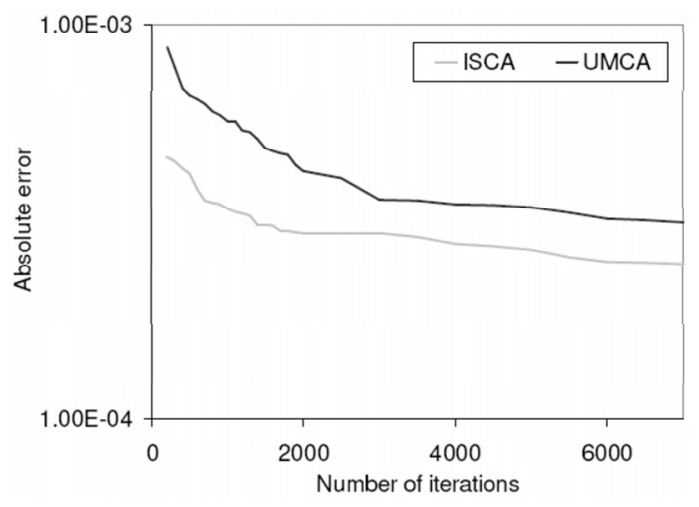

Figure 5. Probability of congestion: absolute error

\section{3) Relative Efficiency}

With last two results we obtain that ISCA is better than UMCA according to the absolute error, but it presents worst performance than UMCA according to computational cost. Table 4 shows the variance of ISCA and UMCA and their respective time cost. Applying formula 11 we obtain that ISCA is around $50 \%$ more efficient than UMCA.

\section{Conclusions}

In this paper new mechanisms to evaluate the Probability of Congestion have been presented in a OPS network with heterogeneous traffic.

The tradeoff between computational cost and accuracy of the estimation has been analyzed. The presented methods overcome previous proposals (CA and ECA) in terms

Table 4. Relative Efficiency

\begin{tabular}{|c|c|c|c|c|}
\hline \multicolumn{2}{|c|}{ Variance } & \multicolumn{2}{c|}{ Time (s) } & \multirow{2}{*}{ Efficiency } \\
\cline { 1 - 4 } ISCA & UMCA & ISCA & UMCA & \\
\hline $8.77 \mathrm{E}-08$ & $1.80 \mathrm{E}-07$ & 0.041224 & 0.029683 & 1.48 \\
\hline $8.73 \mathrm{E}-08$ & $1.29 \mathrm{E}-07$ & 0.051209 & 0.049564 & 1.43 \\
\hline $7.76 \mathrm{E}-08$ & $1.23 \mathrm{E}-07$ & 0.06166 & 0.059556 & 1.53 \\
\hline $7.18 \mathrm{E}-08$ & $1.18 \mathrm{E}-07$ & 0.080968 & 0.069277 & 1.41 \\
\hline $6.22 \mathrm{E}-08$ & $1.04 \mathrm{E}-07$ & 0.093113 & 0.078415 & 1.40 \\
\hline $6.06 \mathrm{E}-08$ & $9.88 \mathrm{E}-08$ & 0.102069 & 0.090259 & 1.44 \\
\hline $5.17 \mathrm{E}-08$ & $8.94 \mathrm{E}-08$ & 0.145694 & 0.122221 & 1.45 \\
\hline
\end{tabular}

of computational cost. The proposed mechanisms (UMCA and ISCA) compute an estimation of the Probability of Congestion. Simulation results show an improvement in performance of our mechanisms in comparison to Enhanced Convolution Approach of 3 orders of magnitude, at the penalty of introducing a small stochastic error in the probability estimation. This error is lower in ISCA case though it has a higher computational cost than UMCA. ISCA, as expected, is more efficient than UMCA in terms of accuracy and computational cost $(50 \%)$.

In our future work Monte Carlo simulations will be carried out in parallel minimizing the added cost and maximizing efficiency.

\section{References}

[1] A. Kaheel et al., "Quality-of-Service Mechanisms in IP-overWDM Networks," IEEE Commun. Mag., pp. 38-43, Dec. 2002.

[2] G. I. Papadimitriou et al., "Optical Switching: Switch Fabrics, Techniques and Architectures," IEEE J. Lightwave Technology, vol. 21, pp. 384-405, Feb. 2003.

[3] M. Duser, et al., "Analysis of a Dynamically WavelengthRouted Optical Burst Switched Network Architecture," IEEE I. Lighiwave Technology, vol. 20, pp. 574-585, Apr. 2002.

[4] N. Boudriga, et al., "Admission Control and Resource Management of Uncertain Duration Traffic in Optical Networks," in Proc. IEEE I0th ICECS, vol. 3, pp. 1018-1021, Dec. 2003.

[5] RACE R2059, "Integrated Communications Management," Technology for ATD, 1991.

[6] F. Davoli et al., "A Two-Level Stochastic Approximation for Admission Control and Bandwidth Allocation," IEEE J. on Select. Areas in Commun., vol. 18, pp. 222-233, Feb. 2000.

[7] J. L. Marzo, "Enhanced Convolution Approach for CAC in ATM Networks, an analytical study and implementation," Ph.D. dissertation, Dept. Electronics, Computers and Automatic Control., Girona Univ., Girona, Spain, 1997.

[8] Q. Wang, V. S. Frost, "Efficient Estimation of Cell Blocking Probability for ATM Systems," IEEE/ACM Trans. on Networking, vol. 1, pp. 230-235, Apr. 1993.

[9] J. A. Freebersyser, J. K. Townsend, "Efficient Simulation of Cell Loss Probability in ATM Networks with Heterogeneous Connection Traffic Descriptors," in Proc. IEEE ICC, 1996.

[10] R. Fabregat et al., "Bandwidth Allocation Based on Real Time Calculations using the Convolution Approach," in Proc. IEEE Globecom, 1994.

[11] R. B. Ash, "Basic Probability Theory," John Wiley \& Sons, Illinois, 1969.

[12] J.M. Hammersley and D.C. Handscomb, "Monte Carlo Methods," Methuen and Co. Lid., 1975. 